\title{
Influence of growth parameters on In-droplet-assisted growth of InAs nanowires on silicon
}

\author{
Ezekiel A. Anyebe ${ }^{1}$
}

Received: 29 May 2017/Accepted: 17 August 2017/Published online: 23 August 2017

(C) The Author(s) 2017. This article is an open access publication

\begin{abstract}
The influence of growth parameters on the morphology and density of InAs nanowires (NWs) grown on bare Si substrates using Indium (In) droplets as catalyst is investigated. By tuning the growth temperature, V/III flux ratio, and growth rate, the diameter and yield of asgrown NWs were controllably manipulated. It is demonstrated that the In-droplet-assisted growth of InAs NWs can only be realized on bare $\mathrm{Si}$ within a relatively narrow growth window of $420-475{ }^{\circ} \mathrm{C}$. Below $420{ }^{\circ} \mathrm{C}$, NWs' growth is kinetically limited, while the highest yield of vertically aligned NWs was obtained at $\sim 450{ }^{\circ} \mathrm{C}$. It is shown that In-catalyzed InAs NWs nucleation can only be realized on $\mathrm{Si}$ at highly As-rich conditions (V/III flux ratio $>50$ ), while the axial growth rate was found to be strongly dependent on the V/III flux ratio. The nucleation and axial growth of In-catalyzed InAs NWs are promoted by a low growth rate, while a high growth rate favors the formation of unwanted parasitic islands.
\end{abstract}

Keywords InAs · Nanowires - Semiconductors ·

Molecular beam epitaxy

\section{Introduction}

Semiconducting nanowires (NWs) have been touted as promising building blocks for applications in photonics and electronic devices (Duan et al. 2003; Tian et al. 2007; Colinge et al. 2010; Tomioka et al. 2012; Wang et al. 2014)

Ezekiel A. Anyebe

ezeanyabe@hotmail.co.uk

1 Federal University of Agriculture, PMB 2373, Makurdi, Nigeria due to their unique properties including superior light absorption, enhanced photo-carrier separation, and epitaxial growth insensitive to lattice mismatch (Wei et al. 2009). In particular, InAs NWs have garnered enormous research interest due to its narrow direct band gap $(0.35 \mathrm{eV})$, small electron effective mass, and high electron mobility of $\sim 30,000 \mathrm{~cm}^{2} / \mathrm{Vs}$ at $300 \mathrm{~K}$ (Wallart et al. 2005; Ihn and Song. 2007) with potential for applications in high-speed electronics and mid-infrared optoelectronic devices (Dimakis et al. 2011). The monolithic integration of NWs with $\mathrm{Si}$ has recently attracted enormous research interest to exploit the low-cost and scalability of the well-established $\mathrm{Si}$ platform as well as the fascinating electronic and optoelectronic properties of NWs. Although, Au is commonly used for NWs nucleation, its use is accompanied with the introduction of unwanted impurities and deep level traps (Allen et al. 2008), which are detrimental to applications in optoelectronic devices (Bar-Sadan et al. 2012). Consequently, there is a paradigm shift towards Au-free technologies including catalyst-free (Wei et al. 2009; Dimakis et al. 2011; Hwang et al. 2015) and droplet-assisted growth (DAG) of NWs (Somaschini et al. 2013; Anyebe et al. 2014; Ermez et al. 2015). Whereas catalyst-free growth is Indium (In) free and requires no catalyst for NWs nucleation, droplet-assisted growth involves the in situ deposition of group-III element droplets (mostly Ga or In seeds) on the substrate prior to growth initiation to function as catalyst for the preferential nucleation of NWs. DAG growth is not only impurity-free and compatible with the complementary metal-oxide semiconductor (CMOS) platform (Glas et al. 2013), it also allows for the precise control of NWs density (Somaschini et al. 2013; Anyebe et al. 2014; Ermez et al. 2015), which is highly essential for applications in functional optoelectronic nanodevices. For instance, the electron mobility (Ford et al. 2009a, b; Abul Khayer and Lake 2010) and light absorption 
(Krogstrup et al. 2013; Heiss et al. 2014), and hence performance of transistors and solar cells, respectively, have been shown to be strongly dependent on the NWs diameter.

It is well established that the size and density of NWs can be controllably manipulated by tuning basic growth precursors (Anyebe et al. 2017; Li et al. 2017). Various growth strategies have been employed for the growth parameter studies of InAs NWs including selective area growth (SAG) (Mandl et al. 2011; Bjoerk et al. 2012), catalyst-free growth on $\mathrm{SiO}_{x}$-coated substrates (Hertenberger et al. 2010; Koblmüller et al. 2010; Madsen et al. 2011), and Au-catalyzed growth (Dayeh et al. 2007; Tchernycheva et al. 2007; Babu and Yoh 2011; Zhang et al. 2016). An investigation of the influence of growth parameters on In-droplet-assisted InAs NWs grown on Si would unravel the conditions for realizing optimal NWs with the highest density and aspect ratio as well as enable for the predictable and reproducible fabrication of high performance, and impurity-free nanoelectronic devices compatible with the CMOS technology. However, despite the significant influence of In-rich conditions (and in effect the predeposition of In droplets) on the NWs geometry (Jung et al. 2014; Zhang et al. 2014) resulting from strong differences in adatom kinetics compared to other growth techniques, little is known about the influence of growth parameters on the morphology and yield of In-catalyzed InAs NWs with the few available reports limited to metal organic chemical vapor deposition (MOCVD) growth on GaAs (Forbes et al. 2010) and InP (Zhang et al. 2013) substrates. Given the significant differences (Cho and Arthur 1975; Finnie and Homma 1999; Arthur 2002; Dubrovskii et al. 2005; Koblmüller et al. 2010) between MOCVD and MBE, it is essential to undertake a growth parameter study of MBE grown In-droplet-assisted InAs NWs on Si. More so, the control of the morphology and yield of InAs NWs directly grown on $\mathrm{Si}$ substrates as a function of growth parameters via an In-assisted technique is yet to be explored despite its enormous potential for the impurity-free integration of III-V NWs on $\mathrm{Si}$, which is, however, not surprising given the very limited report of Incatalyzed InAs NWs growth directly on bare Si (Anyebe et al. 2014). Here, the influence of growth parameters on the morphology and density of InAs nanowires (NWs) grown on bare Si substrates using In droplets is reported. The use of bare $\mathrm{Si}$ substrates minimizes any possible extrinsic contribution.

\section{Experimental details}

InAs NWs were grown on bare $\mathrm{Si}$ (111) substrates by MBE via an In-droplet-assisted growth technique. The Si substrates were first dipped in $12 \%$ hydrofluoric acid solution for about $3 \mathrm{~min}$ to remove the native oxide, then immediately loaded into the MBE system to avoid re-oxidation. The substrates were then thermally outgassed and activated with optimal In droplets (diameter of $\sim 70 \mathrm{~nm}$ and number density of $\sim 6.33 \times 10^{8} \mathrm{~cm}^{-2}$ ) at a temperature of $220{ }^{\circ} \mathrm{C}$ with an In-flux of $2.2 \times 10^{-7} \mathrm{mbar}$ for preferential NWs nucleation (Anyebe et al. 2014). InAs NWs growth was performed by the simultaneous introduction of growth precursors. The influence of growth parameters on NWs geometry and density was then independently investigated, and the surface morphology of the resulting samples was determined by FEI XL30 SFEG scanning electron microscope (SEM).

\section{Results and discussion}

\section{Temperature dependence}

The dependence of InAs NWs morphology and density on growth temperature was first investigated by varying the substrate temperature from 400 to $475{ }^{\circ} \mathrm{C}$, while the In and As fluxes were fixed at $\sim 1.75 \times 10^{-7}$ and $\sim 6.0 \times 10^{-6}$ mbar, respectively, for about $60 \mathrm{~min}$. Figure 1 shows the SEM micrographs of as-grown InAs NWs. Notably, at $400{ }^{\circ} \mathrm{C}$ the growth was dominated by InAs clusters, which suggest a kinetically limited adatom diffusion owing to the significantly low temperature. An increase in growth temperature $\left(G_{\mathrm{T}}\right)$ to $420{ }^{\circ} \mathrm{C}$ resulted in the growth of NWs with an estimated number density of $\sim 9.23 \times 10^{7} \mathrm{~cm}^{-2}$. A further rise in $G_{\mathrm{T}}$ to $450{ }^{\circ} \mathrm{C}$ promoted a slight increase in vertical NWs yield to $\sim 4.78 \times 10^{8} \mathrm{~cm}^{-2}$, which sharply contrasts the complete absence of NWs growth between 440 and $450{ }^{\circ} \mathrm{C}$ in Au- (Tchernycheva et al. 2007; Babu and Yoh 2011) and In-(Forbes et al. 2010) catalyzed InAs NWs growth on InAs and GaAs substrates, respectively. Significantly, compared to the temperature-dependent NWs tapering observed in Au-assisted (Tchernycheva et al. 2007) NWs, all the NWs are vertically aligned with uniform diameter along the growth axis independent of growth temperature. At a high temperature of $475^{\circ} \mathrm{C}$, only a slight decrease in NWs yield was observed, which is attributed to a long adatom diffusion length consistent with previous reports of InAs NW (Dayeh et al. 2007). It is also likely that some of the predeposited In droplets are desorbed from the substrates at such high temperatures leading to the nucleation of fewer NWs (Forbes et al. 2010). This indicates the strong influence of $G_{\mathrm{T}}$ on NWs nucleation probability on Si. The highest yield of vertically aligned NWs was obtained at $\sim 450{ }^{\circ} \mathrm{C}$, while a minimum temperature of $\sim 420{ }^{\circ} \mathrm{C}$ is required for NWs nucleation on bare $\mathrm{Si}$ substrate. This demonstrates that the temperature domain for the nucleation of In-catalyzed InAs NWs on bare Si ranges from 420 to $475{ }^{\circ} \mathrm{C}$, which is slightly narrow when 

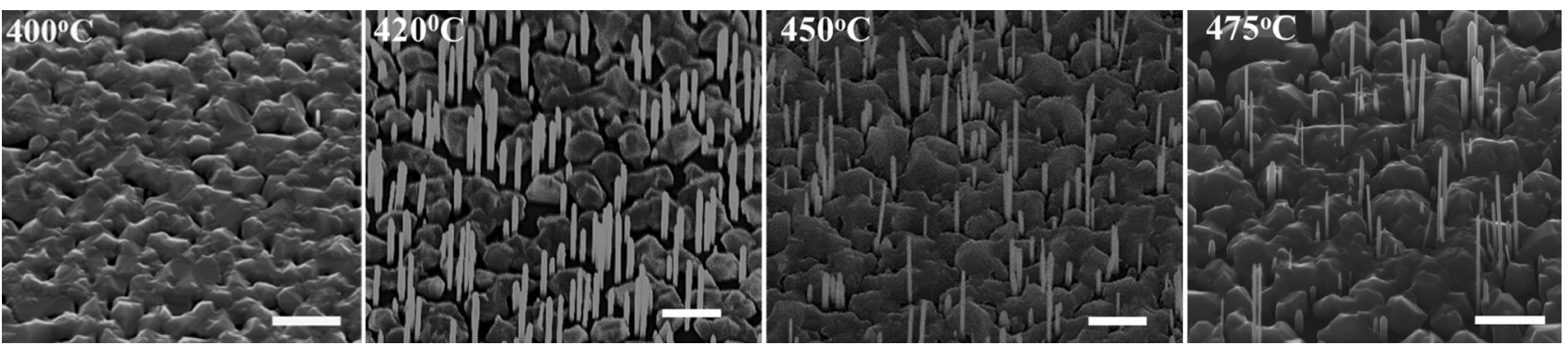

Fig. 1 Tilted SEM images of InAs NWs grown on silicon substrates at fixed In- and As-flux, and varied growth temperatures in the range of $400-475{ }^{\circ} \mathrm{C}$. Tilt angle for all figures is $45^{\circ}$ except at $450{ }^{\circ} \mathrm{C}$ which is $40^{\circ}$. The scale bar for each image is $500 \mathrm{~nm}$

compared to that of MBE (Koblmüller et al. 2010) grown, catalyst-free InAs $\mathrm{NWs}$ on $\mathrm{SiO}_{2} / \mathrm{Si}$ (111) substrates $\left(\sim 400-500{ }^{\circ} \mathrm{C}\right)$. Figure $2 \mathrm{a}$ shows a plot of NWs length $\left(L_{\mathrm{NW}}\right)$ and diameter $\left(D_{\mathrm{NW}}\right)$ as a function of $G_{\mathrm{T}}$. It reveals changes in the axial growth of the NWs; initially $L_{\mathrm{NW}}$ slightly increases with temperature up to $450{ }^{\circ} \mathrm{C}$ after which there was no significant change in $L_{\mathrm{NW}}$ resulting in the realization of maximum $L_{\mathrm{NW}}(1.57 \pm 0.24 \mu \mathrm{m})$ at $450{ }^{\circ} \mathrm{C}$. On the other hand, although thick NWs were realized at $420{ }^{\circ} \mathrm{C}$, the $\mathrm{NWs}$ diameter decreased with increasing temperatures in the range of $420-450{ }^{\circ} \mathrm{C}$ due to the increased adatoms diffusion to the NWs tip in favor of axial growth. This can be interpreted in terms of the temperature-dependent adatom diffusion flux from the substrate to the NWs tip (Dubrovskii et al. 2006; Harmand et al. 2007). The continued decline in diameter beyond $450{ }^{\circ} \mathrm{C}$ is understandable, given the high NWs yield and temperature-dependent In desorption at high temperatures.

\section{Influence of V/III flux ratio}

To investigate the influence of V/III flux ratio on the NWs morphology and yield, the In-flux was fixed at $\sim 1.75 \times 10^{-7}$ mbar, while the V/III flux ratio was tuned from 27 to 55 by varying the As-flux from 4.8 to $9.6 \times 10^{-6}$ mbar at a temperature of $440-500{ }^{\circ} \mathrm{C}$ for a growth duration of $20 \mathrm{~min}$. Figure 3 (top panel) illustrates the variation of NWs morphology and density with V/III flux ratio. As can be seen, NWs nucleation probability is strongly influenced by As-flux. NWs growth was completely inhibited at a relatively low V/III ratio of $\sim 27$ evidenced by the growth of InAs clusters (islands). This is consistent with previous reports (Caroff et al. 2009; Dayeh et al. 2009) and can be understood owing to the fact that group V-rich conditions are required for the nucleation and growth of NWs (Calleja et al. 2007). It is well established that the axial growth of self-catalyzed NWs is highly dependent on the group V flux (Glas et al. 2013; Ramdani et al. 2013; Somaschini et al. 2013; Anyebe et al. 2014; Ermez et al. 2015; Hwang et al. 2015). By slightly raising the V/III ratio to $\sim 51$, a transition to the NWs morphology was induced with the realization of a dense array of NWs (density $=4.23 \times 10^{9} \mathrm{~cm}^{-2}$ ). This demonstrates that Asrich conditions $(\mathrm{As} / \mathrm{In}>50)$ are required for the nucleation and growth of InAs NWs via the droplet epitaxy technique on bare $\mathrm{Si}$, which is consistent with previous report (Babu and Yoh 2011). A small increase in V/III ratio to 55 was, however, accompanied by a decrease in the NWs number density due to the strong dependence of NWs nucleation on In-flux. This observation is in good agreement with a previous study by Dayeh et al. (Dayeh et al. 2007). It was shown that the NW nucleation rate drops as the effective V/III ratio is increased due to the depletion of In from the NW growth sites. Detailed analysis of the SEM images revealed a strong dependence of axial NWs growth on V/III flux ratio (Fig. 2b). $L_{\mathrm{NW}}$ increases monotonically with increasing As-flux in good agreement with a previous report (Babu and Yoh 2011). It has been shown that the elongation rate of self-catalyzed NWs is controlled by the group V flux (Glas et al. 2013; Ramdani et al. 2013). On the other hand, there was no significant change in $D_{\mathrm{NW}}$. This demonstrates that the axial growth of DE grown InAs NWs is limited by As-flux.

\section{Effect of growth rate on nanowire growth}

A series of samples were grown at $440-500{ }^{\circ} \mathrm{C}$ and fixed As-flux of $\sim 6.0 \times 10^{-6}$ mbar, while the $2 \mathrm{D}$ equivalent growth rate was tuned from 0.1 to $0.3 \mu \mathrm{m} / \mathrm{h}$ by varying the In-flux for a growth duration of $60 \mathrm{~min}$ to study the effect of growth rate on InAs NWs growth. Figure 3 (bottom panel) shows the SEM images of InAs NWs grown on Si as a function of growth rate. As can be seen, NWs nucleation significantly decreases with increasing growth rate from 0.1 to $0.2 \mu \mathrm{m} / \mathrm{h}$ at a constant $G_{\mathrm{T}}$. Almost no NWs growth was observed at a growth rate of $0.3 \mu \mathrm{m} / \mathrm{h}$ suggesting NWs growth is promoted by low growth rates. This could likely be associated with the availability of excess In adatoms possibly as a direct consequence of contributions from the pre-deposited In droplets. Excess accumulation of In and surface growths imposes certain restrictions in the collection area thereby inhibiting adatom mobility and leading to 

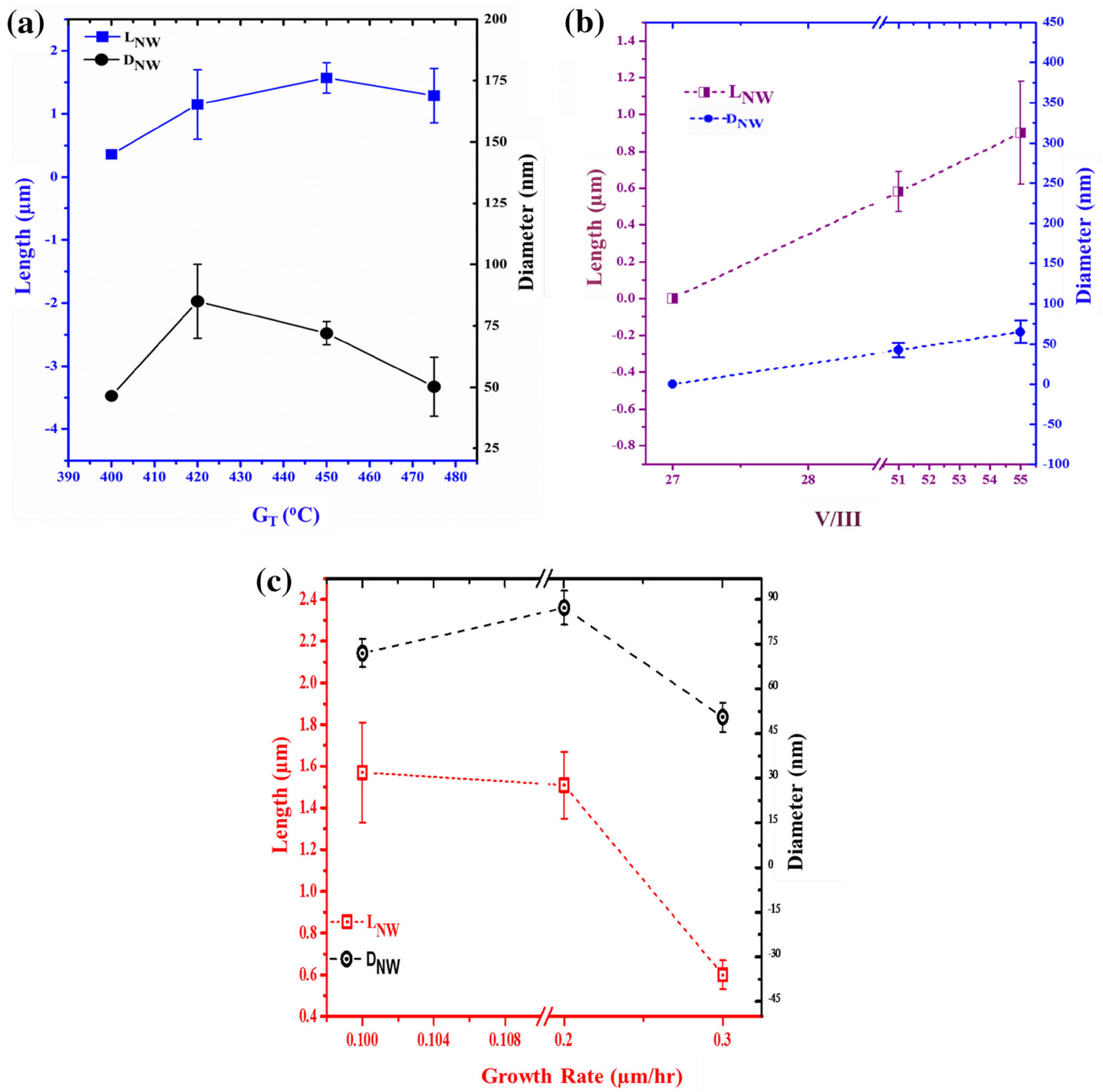

Fig. 2 Plot of InAs nanowires length $\left(L_{\mathrm{NW}}\right)$ and diameter $\left(D_{\mathrm{NW}}\right)$ on bare silicon substrates as a function of a growth temperature $\left(G_{\mathrm{T}}\right)$, b V/III flux ratio and $\mathbf{c}$ growth rate, respectively

a decline in NWs nucleation probability and number density. The high volume of InAs clusters (islands) at a growth rate of $0.3 \mu \mathrm{m} / \mathrm{h}$ provides clear evidence of the presence of excess surface growth on the substrate (Persson et al. 2007). This demonstrates that the nucleation and yield of InAs NWs is promoted by a low growth rate and in effect low In-flux (high V/III flux ratio), which is consistent with the observed dependence of NWs nucleation on the As-flux (as explained in the previous section). Conversely, a high growth rate promotes InAs Islands (clusters) growth.
Figure 2c shows the dependence of $L_{\mathrm{NW}}$ and $D_{\mathrm{NW}}$ on growth rate. There was no clear change in $L_{\mathrm{NW}}$ and $D_{\mathrm{NW}}$ with increasing growth rate from 0.1 to $0.2 \mu \mathrm{m} / \mathrm{h}$. However, when a growth rate of $0.3 \mu \mathrm{m} / \mathrm{h}$ was utilized, $L_{\mathrm{NW}}$ decreased by over two orders of magnitude and $D_{\mathrm{NW}}$ reduced by $\sim 2 \times$. This is in sharp contrast to the reported enhancement in axial growth rate and density of catalystfree InAs NWs on Si (Dimakis et al. 2011). The observed trend can be explained by the significant decrease in the available adatom at the growth front due to the suppression 

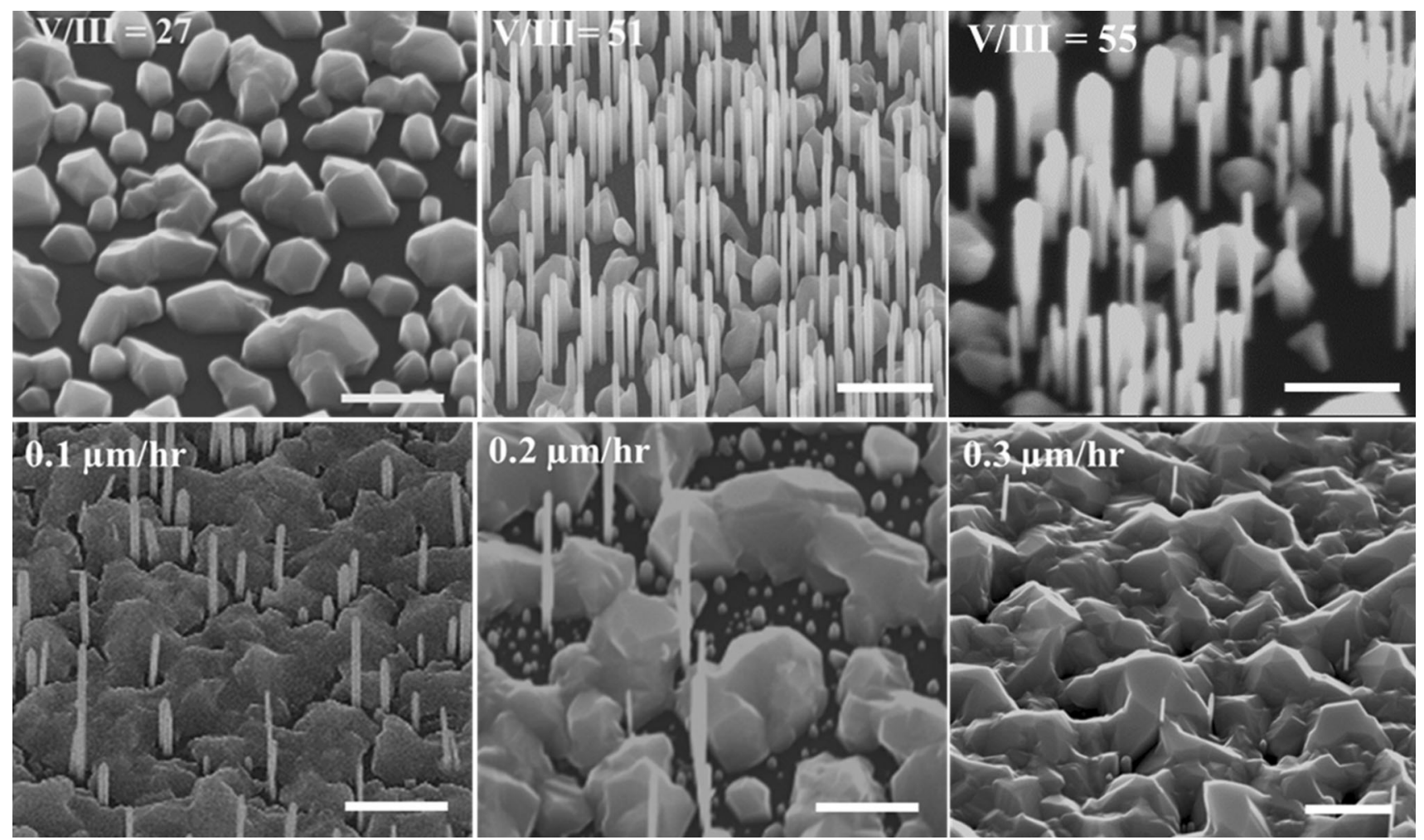

Fig. $345^{\circ}$ tilted SEM images of InAs nanowires grown on Si as a function of various V/III flux ratio (top panel) and growth rates (bottom panel) with scale bars of $500 \mathrm{~nm}$ and $1 \mu \mathrm{m}$, respectively

of adatom diffusion by the surface Islands. This clearly indicates that high growth rate does not favor the axial growth and yield of DE grown InAs NWs rather, it promotes the formation of Islands.

\section{Conclusion}

It has been shown that the morphology and density of NWs can be independently controlled by tuning the basic growth parameters such as growth temperature, growth rate, and $\mathrm{V} / \mathrm{III}$ flux ratio. A relatively narrow temperature window of $420-475{ }^{\circ} \mathrm{C}$ has been identified for InAs NWs growth on bare $\mathrm{Si}$. No NWs growth was realized at temperatures below $420{ }^{\circ} \mathrm{C}$, while the highest yield of vertically aligned NWs was obtained at $\sim 450{ }^{\circ} \mathrm{C}$. It is shown that In-catalyzed InAs NWs nucleation can only be realized on $\mathrm{Si}$ at highly As-rich conditions (V/III $>50$ ), while the axial growth rate was found to be strongly influenced by the V/III flux ratio. A high yield of vertically aligned InAs NWs is promoted by low growth rate on bare $\mathrm{Si}$, while a high growth rate favors the formation of Island.

Acknowledgements The author gratefully acknowledge Lancaster University, UK, for providing MBE Facility for this study. The author thanks Q.D. Zhuang of Lancaster University, UK for support in MBE epitaxy, Mohana K. Rajpalke and T.D. Veal of, University of
Liverpool for assistance in electron microscopy characterization. This work was supported by Tertiary Education Trust Fund (TETFund), Nigeria.

Open Access This article is distributed under the terms of the Creative Commons Attribution 4.0 International License (http:// creativecommons.org/licenses/by/4.0/), which permits unrestricted use, distribution, and reproduction in any medium, provided you give appropriate credit to the original author(s) and the source, provide a link to the Creative Commons license, and indicate if changes were made.

\section{References}

Abul Khayer M, Lake RK (2010) Diameter dependent performance of high-speed, low-power InAs nanowire field-effect transistors. J Appl Phys 107:014502

Allen JE, Hemesath ER, Perea DE et al (2008) High-resolution detection of Au catalyst atoms in Si nanowires. Nat Nanotechnol 3:168-173

Anyebe EA, Zhuang Q, Sanchez A et al (2014) Self-catalysed growth of InAs nanowires on bare Si substrates by droplet epitaxy. Rapid Res Lett 8:658-662

Anyebe EA, Sandall I, Jin ZM et al (2017) Optimization of selfcatalyzed InAs nanowires on flexible graphite for photovoltaic infrared photodetectors. Sci Rep 7:46110

Arthur JR (2002) Molecular beam epitaxy. Surf Sci 500:189-217

Babu JB, Yoh K (2011) Effect of As/In-flux on the growth of InAs nanowire by molecular beam epitaxy. J Cryst Growth 323:301-303

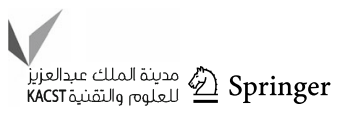


Bar-Sadan M, Barthel J, Shtrikman H, Houben L (2012) Direct imaging of single $\mathrm{Au}$ atoms within GaAs nanowires. Nano Lett 12:2352-2356

Bjoerk MT, Schmid H, Breslin CM et al (2012) InAs nanowire growth on oxide-masked $\langle 111\rangle$ silicon. J Cryst Growth 344:31-37

Calleja E, Ristic J, Fernandez-Garrido S et al (2007) Growth, morphology, and structural properties of group-III-nitride nanocolumns and nanodisks. Phys Status Solidi B-Basic Solid State Phys 244:2816-2837

Caroff P, Messing ME, Borg BM et al (2009) InSb heterostructure nanowires: MOVPE growth under extreme lattice mismatch. Nanotechnology 20:495606

Cho AY, Arthur JR (1975) Molecular beam epitaxy. Progress in solid state chemistry. Pergamon, New York, pp 157-191

Colinge JP, Lee C-W, Afzalian A et al (2010) Nanowire transistors without junctions. Nat Nanotechnol 5:225-229

Dayeh SA, Yu ET, Wang D (2007) III-V nanowire growth mechanism: V/III ratio and temperature effects. Nano Lett 7:2486-2490

Dayeh SA, Yu ET, Wang DL (2009) Surface diffusion and substratenanowire adatom exchange in InAs nanowire growth. Nano Lett 9:1967-1972

Dimakis E, Lähnemann J, Jahn U et al (2011) Self-assisted nucleation and vapor-solid growth of InAs nanowires on bare $\mathrm{Si}(111)$. Cryst Growth Des 11:4001-4008

Duan X, Huang Y, Agarwal R, Lieber CM (2003) Single-nanowire electrically driven lasers. Nature 421:241-245

Dubrovskii VG, Cirlin GE, Soshnikov IP et al (2005) Diffusioninduced growth of GaAs nanowhiskers during molecular beam epitaxy: theory and experiment. Phys Rev B 71:205325

Dubrovskii VG, Sibirev NV, Cirlin GE et al (2006) Theoretical analysis of the vapor-liquid-solid mechanism of nanowire growth during molecular beam epitaxy. Phys Rev E 73:21603-21610

Ermez S, Jones EJ, Crawford SC, Gradecak S (2015) Self-seeded growth of GaAs nanowires by metal-organic chemical vapor deposition. Cryst Growth Des 15:2768-2774

Finnie P, Homma Y (1999) Maskless selective area molecular beam epitaxy of semiconductors and metals using atomic step networks on silicon. J Cryst Growth 201:604-609

Forbes D, Hubbard S, Raffaelle R, McNatt JS (2010) Au-catalyst-free epitaxy of InAs nanowires. J Cryst Growth 312:1391-1395

Ford AC, Ho JC, Chueh Y-L et al (2009a) Monolayer doping and diameter-dependent electron mobility assessment of nanowires. IEEE Int Conf Integr Circuit Des Technol Proc 2009:223-227

Ford AC, Ho JC, Chueh Y-L et al (2009b) Diameter-dependent electron mobility of InAs nanowires. Nano Lett 9:360-365

Glas F, Ramdani MR, Patriarche G, Harmand JC (2013) Predictive modeling of self-catalyzed III-V nanowire growth. Phys Rev B 88:195304-1953014

Harmand JC, Tchernycheva M, Patriarche G et al (2007) GaAs nanowires formed by Au-assisted molecular beam epitaxy: effect of growth temperature. J Cryst Growth 301:853-856

Heiss M, Russo-Averchi E, Dalmau-Mallorquí A et al (2014) III-V nanowire arrays: growth and light interaction. Nanotechnology $25: 14015$

Hertenberger S, Rudolph D, Bichler M et al (2010) Growth kinetics in position-controlled and catalyst- free InAs nanowire arrays on $\mathrm{Si}(111)$ grown by selective area molecular beam epitaxy. J Appl Phys 108:114316-114317
Hwang JW, Kim B-K, Lee SJ et al (2015) Catalyst-free heteroepitaxial growth of very long InAs nanowires on Si. Curr Appl Phys 15:S35-S39

Ihn S-G, Song J-I (2007) InAs nanowires on Si substrates grown by solid source molecular beam epitaxy. Nanotechnology 18:355603

Jung K, Mohseni PK, Li X (2014) Ultrathin InAs nanowire growth by spontaneous Au nanoparticle spreading on indium-rich surfaces. Nanoscale 6:15293-15300

Koblmüller G, Hertenberger S, Vizbaras K et al (2010) Self-induced growth of vertical free-standing InAs nanowires on $\mathrm{Si}$ (111) by molecular beam epitaxy. Nanotechnology 21:365602

Krogstrup P, Jorgensen HI, Heiss M et al (2013) Single-nanowire solar cells beyond the Shockley-Queisser limit. Nat Photonics 7:306-310

Li B, Yan X, Zhang X, Ren X (2017) Self-catalyzed growth of InAs nanowires on InP substrate. Nanoscale Res Lett 12:34

Madsen MH, Aagesen M, Krogstrup P et al (2011) Influence of the oxide layer for growth of self-assisted InAs nanowires on $\mathrm{Si}(111)$. Nanoscale Res Lett 6:516

Mandl B, Dey AW, Stangl J et al (2011) Self- seeded, positioncontrolled InAs nanowire growth on $\mathrm{Si}$ : a growth parameter study. J Cryst Growth 334:51-56

Persson AI, Froberg LE, Jeppesen S et al (2007) Surface diffusion effects on growth of nanowires by chemical beam epitaxy. J Appl Phys 101:34313-34316

Ramdani MR, Harmand JC, Glas F et al (2013) Arsenic pathways in self-catalyzed growth of GaAs nanowires. Cryst Growth Des 13:91-96

Somaschini C, Bietti S, Trampert A et al (2013) Control over the number density and diameter of $\mathrm{GaAs}$ nanowires on $\mathrm{Si}(111)$ mediated by droplet epitaxy. Nano Lett 13:3607-3613

Tchernycheva M, Travers L, Patriarche G et al (2007) Au- assisted molecular beam epitaxy of InAs nanowires: growth and theoretical analysis. J Appl Phys 102:94313-94318

Tian Y, Huang R, Wang Y et al (2007) New self-aligned silicon nanowire transistors on bulk substrate fabricated by epi-free compatible CMOS technology: Process integration, experimental characterization of carrier transport and low frequency noise. In: Electron Devices Meeting, IEDM. IEEE International, pp 895-898

Tomioka K, Yoshimura M, Fukui T (2012) A III-V nanowire channel on silicon for high-performance vertical transistors. Nature 488:189-192

Wallart X, Lastennet J, Vignaud D, Mollot F (2005) Performances and limitations of InAs/InAlAs metamorphic heterostructures on InP for high mobility devices. Appl Phys Lett 87:43504

Wang X, Tong J, Chen X et al (2014) Highly ordered GaN-based nanowire arrays grown on patterned (100) silicon and their optical properties. Chem Commun 50:682-684

Wei W, Bao XY, Soci C et al (2009) Direct heteroepitaxy of vertical InAs nanowires on $\mathrm{Si}$ substrates for broad band photovoltaics and photodetection. Nano Lett 9:2926-2934

Zhang G, Sasaki S, Tateno K et al (2013) Au-free InAs nanowires grown in In-particle-assisted vapor-liquid-solid mode: growth, structure, and electrical property. AIP Adv 3:52107

Zhang Z, Lu Z, Xu H et al (2014) Structure and quality controlled growth of InAs nanowires through catalyst engineering. Nano Res 7:1640-1649

Zhang Z, Chen P-P, Lu W, Zou J (2016) Defect-free thin InAs nanowires grown using molecular beam epitaxy. Nanoscale 8:1401-1406 